\title{
Periodic solutions for a class of second order Hamiltonian systems with $p(t)$-Laplacian
}

\section{Shengui Zhang*}

\section{"Correspondence:}

zhangshengui315@163.com Department of Mathematics, Sichuan University, Chengdu, Sichuan 610064, P.R. China

\begin{abstract}
By means of a variational analysis and the theory of variable exponent Sobolev spaces, we study the existence of periodic solutions for a class of nonlocal Hamiltonian systems with $p(t)$-Laplacian. Some new solvability conditions of periodic solutions are obtained; this unifies and generalizes some of the recent corresponding results in the literature.
\end{abstract}

MSC: $34 \mathrm{C} 37 ; 58 \mathrm{E} 05 ; 70 \mathrm{H} 05$

Keywords: periodic solution; nonlocal $p(t)$-Laplacian Hamiltonian systems; the variable exponent Sobolev spaces; critical point theory

\section{Introduction}

In this paper, we consider the existence of periodic solutions of the following $p(t)$ Laplacian Hamiltonian systems:

$$
\left\{\begin{array}{l}
M\left(\int_{0}^{T} \frac{|\dot{u}(t)|^{p(t)}}{p(t)} d t\right) \frac{d}{d t}\left(|\dot{u}(t)|^{p(t)-2} \dot{u}(t)\right)=\nabla F(t, u(t)), \quad \text { a.e. } t \in[0, T] \\
u(0)-u(T)=\dot{u}(0)-\dot{u}(T)=0
\end{array}\right.
$$

where $T>0$ and $\nabla F(t, u)$ denotes its gradient with respect to the second variable, that is,

$$
\begin{aligned}
& \nabla F(t, u)=\left(\frac{\partial F}{\partial u_{1}}, \ldots, \frac{\partial F}{\partial u_{N}}\right), \\
& u(t)=\left(u_{1}(t), \ldots, u_{N}(t)\right) .
\end{aligned}
$$

In the sequel, we assume that the variable exponent $p(t) \in C\left([0, T] ; \mathbb{R}^{+}\right), p(t)=p(t+T)$, and

$$
1<p^{-}:=\min _{0 \leq t \leq T} p(t) \leq p^{+}:=\max _{0 \leq t \leq T} p(t)<+\infty
$$

Moreover, we suppose that $M(s): \mathbb{R}^{+} \rightarrow \mathbb{R}^{+}$are continuous functions and $F:[0, T] \times$ $\mathbb{R}^{N} \rightarrow \mathbb{R}$ satisfies the following assumption:

(c) The Author(s) 2016. This article is distributed under the terms of the Creative Commons Attribution 4.0 International License (http://creativecommons.org/licenses/by/4.0/), which permits unrestricted use, distribution, and reproduction in any medium, provided you give appropriate credit to the original author(s) and the source, provide a link to the Creative Commons license, and indicate if changes were made. 
(A) $F(t, x)$ is measurable in $t$ for each $x \in \mathbb{R}^{N}$ and continuously differentiable in $x$ for a.e. $t \in[0, T]$, and there exist $a \in C\left(\mathbb{R}^{+}, \mathbb{R}^{+}\right), b \in L^{1}\left([0, T] ; \mathbb{R}^{+}\right)$such that

$$
|F(t, x)| \leq a(|x|) b(t), \quad|\nabla F(t, x)| \leq a(|x|) b(t)
$$

for all $x \in \mathbb{R}^{N}$ and a.e. $t \in[0, T]$.

Throughout this paper, we denote the usual norm and the usual inner product in $\mathbb{R}^{N}$, respectively, by $|\cdot|$ and $(\cdot, \cdot)$.

Especially, when $p(t) \equiv 2$ and $M(s) \equiv 1$, problem (1.1) becomes the well-known second order Hamiltonian system

$$
\left\{\begin{array}{l}
\ddot{u}(t)=\nabla F(t, u(t)), \quad \text { a.e. } t \in[0, T], \\
u(0)-u(T)=\dot{u}(0)-\dot{u}(T)=0 .
\end{array}\right.
$$

In the monograph [1], Mawhin and Willem investigated the existence of periodic solutions for problem (1.3) and obtained a series of results. During the past two decades, inspired by [1] the existence of periodic solutions for systems (1.3) has been studied via critical point theory; for example, see [2-10]. In some recent work, a potential $F(t, x)$ is the sum of an autonomous potential $F_{1}(u)$ and a non-autonomous potential $F_{2}(t, u)$, where the autonomous term satisfies a directional bounded on the gradient, that is, there exists a positive constant $r$ such that

$$
\left(\nabla F_{1}(x)-\nabla F_{1}(y), x-y\right) \leq r|x-y|^{2}
$$

for all $x, y \in \mathbb{R}^{N}$, and the gradient of non-autonomous term is bounded, sublinear or $F_{1}(t, x)$ grows to $\infty$ as $|x| \rightarrow \infty$; see $[3,5,7]$.

When the nonlocal coefficient $M(s) \equiv 1$, problem (1.1) becomes

$$
\left\{\begin{array}{l}
\frac{d}{d t}\left(|\dot{u}(t)|^{p(t)-2} \dot{u}(t)\right)=\nabla F(t, u(t)), \quad \text { a.e. } t \in[0, T] \\
u(0)-u(T)=\dot{u}(0)-\dot{u}(T)=0 .
\end{array}\right.
$$

The operator $\frac{d}{d t}\left(|\dot{u}(t)|^{p(t)-2} \dot{u}(t)\right)$ is said to be $p(t)$-Laplacian. It is well known that problems with the $p(t)$-Laplacian operator possess more complicated nonlinearities than the constant case. Nonlinear problems with a variable exponent Laplacian arise in lots of mathematical physics contexts, such as this type of problems can be applied to describe the physical phenomena with 'pointwise different properties'. The study of boundary problems with $p(t)$-Laplacian is now an active area of research. In particular, by using critical point theory, considerable attention has been paid to the periodic solutions of problem (1.4); see [11-21].

Since the equations in (1.1) contain an integral over $[0, T]$, this problem is no longer a pointwise identity. This type of problems are often called Kirchhoff-type or nonlocal problems, they arise in the description of nonlinear vibrations of an elastic string. Chipot and Lovat [22] pointed out that this type of problems can be used to model a spreading process of a particular species within the domain, where $u$ is its population density.

Recently, a lot of researchers have investigated $p(x)$-Kirchhoff-type equations with Dirichlet or Neumann boundary condition; see [23-27]. However, to the best of our knowledge, there are very scarce papers discussing periodic solutions for system (1.1). 
Motivated by the results mentioned above, we first prove that problem (1.1) has at least one periodic solution. The periodic solutions which we get appear either as saddle points or as minimizers of the energy functional associated to problem (1.1). Next, based on the three critical points theorem due to Brezis and Nirenberg, we will obtain the existence of at least two distinct periodic solutions of the problem (1.1). Finally, when the potential energy term is even, we are going to study the existence of infinitely many periodic solutions of problem (1.1).

\section{Preliminaries}

Now, we recall the properties of the space $W_{T}^{1, p(t)}$ listed for the convenience of the reader. In this section, the definitions and lemmas can be found in $[12,13,18]$.

Definition $2.1([12])$ Let $p(t) \in C\left([0, T] ; \mathbb{R}^{+}\right)$, and $p(t)$ satisfy $(1.2)$. Define

$$
L^{p(t)}\left([0, T] ; \mathbb{R}^{N}\right)=\left\{\left.u \in L^{1}\left([0, T] ; \mathbb{R}^{N}\right)\left|\int_{0}^{T}\right| u\right|^{p(t)} d t<\infty\right\}
$$

with the norm

$$
|u|_{p(t)}=\inf \left\{\lambda>\left.0\left|\int_{0}^{T}\right| \frac{u}{\lambda}\right|^{p(t)} d t \leq 1\right\}
$$

Define

$$
C_{T}^{\infty}=C_{T}^{\infty}\left(\mathbb{R}, \mathbb{R}^{N}\right)=\left\{u \in C^{\infty}\left(\mathbb{R}, \mathbb{R}^{N}\right) \mid u \text { is } T \text {-periodic }\right\}
$$

Definition $2.2([12])$ Let $u \in L^{1}\left([0, T] ; \mathbb{R}^{N}\right)$ and $v \in L^{1}\left([0, T] ; \mathbb{R}^{N}\right)$. If

$$
\int_{0}^{T} v \phi d t=-\int_{0}^{T} u \phi^{\prime} d t, \quad \forall \phi \in C_{T}^{\infty}
$$

then $v$ is called the $T$-weak derivative of $u$ and is denoted by $\dot{u}$.

Definition 2.3 ([12]) Define

$$
W_{T}^{1, p(t)}=\left\{u:[0, T] \rightarrow \mathbb{R}^{N} \mid u \in L^{p(t)}\left([0, T] ; \mathbb{R}^{N}\right), \dot{u} \in L^{p(t)}\left([0, T] ; \mathbb{R}^{N}\right), u(0)=u(T)\right\}
$$

with the norm

$$
\|u\|=|u|_{p(t)}+|\dot{u}|_{p(t)}
$$

When $p^{-}=\min _{0 \leq t \leq T} p(t)>1, W_{T}^{1, p(t)}$ is a reflexive Banach space.

For $u \in W_{T}^{1, p(t)}$, we write

$$
\|u\|=|\bar{u}|+|\dot{u}|_{p(t)}
$$

then $\|\cdot\|$ is an equivalent norm on $W_{T}^{1, p(t)}$, where $\bar{u}=\frac{1}{T} \int_{0}^{T} u(t) d t$. 
For $u \in W_{T}^{1, p(t)}$ we can write $u(t)=\bar{u}+\tilde{u}(t)$. Denoting

$$
\widetilde{W}_{T}^{1, p(t)}=\left\{u \in W_{T}^{1, p(t)} \mid \int_{0}^{T} u(t) d t=0\right\}
$$

then $W_{T}^{1, p(t)}=\widetilde{W}_{T}^{1, p(t)} \oplus \mathbb{R}^{N}$.

Lemma 2.1 ([12]) If we denote

$$
\rho(u)=\int_{0}^{T}|u(t)|^{p(t)} d t, \quad \forall u \in L^{p(t)}
$$

then

(i) $|u|_{p(t)}<1(=1 ;>1) \Leftrightarrow \rho(u)<1(=1 ;>1)$;

(ii) $|u|_{p(t)}>1 \Rightarrow|u|_{p(t)}^{p^{-}} \leq \rho(u) \leq|u|_{p(t)}^{p^{+}} ;|u|_{p(t)}<1 \Rightarrow|u|_{p(t)}^{p^{+}} \leq \rho(u) \leq|u|_{p(t)}^{p^{-}}$;

(iii) $|u|_{p(t)} \rightarrow 0 \Leftrightarrow \rho(u) \rightarrow 0$; $|u|_{p(t)} \rightarrow \infty \Leftrightarrow \rho(u) \rightarrow \infty$.

Applying Lemma 2.1, one has the following.

Lemma $2.2([12])$ For all $\tilde{u} \in \widetilde{W}_{T}^{1, p(t)}$, we have

$$
\begin{aligned}
& \|\tilde{u}\|>1 \quad \Rightarrow \quad\|\tilde{u}\|^{p^{-}} \leq \int_{0}^{T}|\dot{u}(t)|^{p(t)} d t \leq\|\tilde{u}\|^{p^{+}} ; \\
& \|\tilde{u}\|<1 \quad \Rightarrow \quad\|\tilde{u}\|^{p^{+}} \leq \int_{0}^{T}|\dot{u}(t)|^{p(t)} d t \leq\|\tilde{u}\|^{p^{-}} ; \\
& \|\tilde{u}\|=1 \quad \Rightarrow \quad \int_{0}^{T}|\dot{u}(t)|^{p(t)} d t=1 .
\end{aligned}
$$

Lemma 2.3 ([18]) For all $\tilde{u} \in \widetilde{W}_{T}^{1, p(t)}$, there exist constants $C_{i}>0, i=0,1,2$, such that

$$
\begin{aligned}
& \|\tilde{u}\|_{\infty}:=\max _{0 \leq t \leq T}|u(t)| \leq 2 C_{0}\left(\int_{0}^{T}|\dot{u}(t)|^{p(t)} d t\right)^{\frac{1}{p^{-}}}+C_{1}, \\
& \|\tilde{u}\| \leq C_{2}\left[\left(\int_{0}^{T}|\dot{u}(t)|^{p(t)} d t\right)^{\frac{1}{p^{-}}}+1\right] .
\end{aligned}
$$

Lemma 2.4 ([18]) For all $u \in W_{T}^{1, p(t)}, \bar{u}=\frac{1}{T} \int_{0}^{T} u(t) d t$, then

$$
\|u\| \rightarrow \infty \quad \Rightarrow \quad \int_{0}^{T}|\dot{u}(t)|^{p(t)} d t+|\bar{u}| \rightarrow+\infty
$$

Lemma 2.5 ([12]) There is a continuous embedding $W_{T}^{1, p(t)} \hookrightarrow C\left([0, T] ; \mathbb{R}^{N}\right)$, when $p^{-}>1$, the embedding is compact.

By Lemma 2.3 and (1.2), there exists $d_{0}>0$, such that

$$
\|u\|_{\infty}:=\max _{0 \leq t \leq T}|u(t)| \leq d_{0}\|u\|, \quad \forall u \in W_{T}^{1, p(t)}
$$


Lemma 2.6 ([13]) $J^{\prime}$ is a bounded linear functional and a mapping of $\left(S_{+}\right)$on $W_{T}^{1, p(t)}$, that is, if $u_{n} \rightarrow u$ weakly in $W_{T}^{1, p(t)}$ and $\limsup _{n \rightarrow \infty}\left(J^{\prime}\left(u_{n}\right)-J^{\prime}(u), u_{n}-u\right) \leq 0$, then $\left\{u_{n}\right\}$ has a convergent subsequence, where $J^{\prime}$ is given by $\left\langle J^{\prime}(u), v\right\rangle=\int_{0}^{T}\left(|\dot{u}(t)|^{p(t)-2} \dot{u}(t), \dot{v}(t)\right) d t$.

\section{Main results}

For our results, the following conditions are assumed for the function $M(s)$.

$\left(\mathrm{M}_{0}\right)$ There exists a constant $m_{0}>0$ such that inf $\mathrm{in}_{s \in \mathbb{R}^{+}} M(s) \geq m_{0}$.

$\left(\mathrm{M}_{1}\right)$ For all $s \in[0,+\infty)$, let $\widehat{M}(s):=\int_{0}^{s} M(\sigma) d \sigma$ such that $\widehat{M}(s) \leq M(s) s$.

We assume that $F(t, x)=F_{1}(t, x)+F_{2}(x)$ satisfies the following hypotheses:

$\left(\mathrm{H}_{1}\right)$ There exist constants $K_{i}>0, i=1,2,3, \alpha \in\left[0, p^{-}-1\right)$ and a function $h \in C([0, \infty)$, $[0, \infty))$, with the properties:

(i) $h(s) \leq h(t), \forall s \leq t, s, t \in[0, \infty)$.

(ii) $h(s+t) \leq K_{0}(h(s)+h(t)), \forall s, t \in[0, \infty)$.

(iii) $0 \leq h(s) \leq K_{1} s^{\alpha}+K_{2}, \forall s, t \in[0, \infty)$.

(iv) $h(s) \rightarrow \infty$ as $s \rightarrow \infty$.

$\left(\mathrm{H}_{2}\right)$ There exist $f \in L^{1}\left(0, T ; \mathbb{R}^{+}\right)$and $g \in L^{1}\left(0, T ; \mathbb{R}^{+}\right)$such that

$$
\left|\nabla F_{1}(t, x)\right| \leq f(t) h(|x|)+g(t)
$$

for all $x \in \mathbb{R}^{N}$ and a.e. $t \in[0, T]$.

$\left(\mathrm{H}_{3}\right)$ There exist constants $r>0$ and $\gamma \in\left[0, p^{-}\right)$such that

$$
\left(\nabla F_{2}(x)-\nabla F_{2}(y), x-y\right) \geq-r|x-y|^{\gamma}
$$

for all $x, y \in \mathbb{R}^{N}$.

$\left(\mathrm{H}_{4}\right)$ There exists a constant $\theta>1$ such that

$$
\liminf _{|x| \rightarrow \infty} \frac{1}{h^{q^{+}(|x|)}} \int_{0}^{T} F(t, x) d t>\frac{1}{q^{+}}\left(2 K_{0} C_{0} \int_{0}^{T} f(t) d t\right)^{q^{+}}\left(\frac{\theta p^{+}}{m_{0} p^{-}}\right)^{\frac{q^{+}}{p^{-}}}
$$

where $\frac{1}{p^{-}}+\frac{1}{q^{+}}=1$ and $C_{0}$ is a positive constant which satisfies the inequality (2.1).

First, using the least action principle in critical point theory, we get the first main result.

Theorem 3.1 Suppose that assumption (A), conditions $\left(\mathrm{M}_{0}\right)-\left(\mathrm{M}_{1}\right)$ and $\left(\mathrm{H}_{1}\right)-\left(\mathrm{H}_{4}\right)$ hold, then problem (1.1) has at least one T-periodic solution which minimizes the functional $\Phi$ in $W_{T}^{1, p(t)}$ given by

$$
\Phi(u)=\widehat{M}\left(\int_{0}^{T} \frac{|\dot{u}(t)|^{p(t)}}{p(t)} d t\right)+\int_{0}^{T} F(t, u(t)) d t
$$

for $u \in W_{T}^{1, p(t)}$

Next, we obtain the second main result by using the saddle point theorem. 
$\left(\mathrm{H}_{5}\right)$ There exist constants $\beta \in\left[0, p^{-}\right)$and $\mu>0$ such that

$$
\left(\nabla F_{2}(x)-\nabla F_{2}(y), x-y\right) \leq \mu|x-y|^{\beta}
$$

for all $x, y \in \mathbb{R}^{N}$.

$\left(\mathrm{H}_{6}\right)$ There exists a constant $\theta>1$, let

$$
K=\frac{1}{q^{+}}\left(\frac{1}{p^{-}}+1\right) \frac{\theta p^{+}+1}{\theta p^{+}-1}\left(2 K_{0} C_{0} \int_{0}^{T} f(t) d t\right)^{q^{+}}\left(\frac{\theta p^{+}}{m_{0} p^{-}}\right)^{\frac{q^{+}}{p^{-}}}
$$

such that

$$
\limsup _{|x| \rightarrow \infty} \frac{1}{h^{q^{+}}(|x|)} \int_{0}^{T} F(t, x) d t<-K
$$

Theorem 3.2 Suppose that assumption (A), conditions $\left(\mathrm{M}_{0}\right)-\left(\mathrm{M}_{1}\right),\left(\mathrm{H}_{1}\right)-\left(\mathrm{H}_{3}\right),\left(\mathrm{H}_{5}\right)$, and $\left(\mathrm{H}_{6}\right)$ hold, then problem (1.1) has at least one T-periodic solution in $W_{T}^{1, p(t)}$.

Remark 3.3 The nonlocal coefficient $M\left(\int_{0}^{T} \frac{|\dot{u}(t)|^{p(t)}}{p(t)} d t\right)$ represent Kirchhoff dissipative terms. A typical example is the following:

$$
M(s)=1+k s^{m}
$$

for all $s \in[0,+\infty)$, where $k$ and $m$ are positive constants. Then $M(t)$ satisfies $\left(\mathrm{M}_{0}\right)-\left(\mathrm{M}_{1}\right)$. In fact,

$$
M(s)=1+k s^{m} \geq 1>0
$$

and

$$
\widehat{M}(s)=\int_{0}^{s} M(\sigma) d \sigma=\int_{0}^{s}\left(1+k \sigma^{m}\right) d \sigma \leq \int_{0}^{s}\left(1+k s^{m}\right) d \sigma=M(s) s .
$$

Remark 3.4 When $M(s) \equiv 1, p(t) \equiv 2$, condition $\left(\mathrm{H}_{1}\right)$ and $\left(\mathrm{H}_{2}\right)$ were introduced in [2], which is an extension of the usual sublinear growth condition, i.e. there exist $\alpha \in[0,1)$, $f \in L^{1}\left(0, T ; \mathbb{R}^{+}\right)$and $g \in L^{1}\left(0, T ; \mathbb{R}^{+}\right)$, such that

$$
\left|\nabla F_{1}(t, x)\right| \leq f(t)|x|^{\alpha}+g(t)
$$

for all $x \in \mathbb{R}^{N}$ and a.e. $t \in[0, T]$. From condition $\left(\mathrm{H}_{1}\right)$ and $\left(\mathrm{H}_{2}\right)$, we can see that the nonlinearity $\nabla F_{1}(t, x)$ grows sightly slower that $|x|^{\alpha}$. If $M(s) \equiv 1$ and $p(t)=\sin \frac{2 \pi t}{T}+5$, then $m_{0}=1$, $p^{+}=6, p^{-}=4$, and $q^{+}=\frac{4}{3}$. Consider the functions

$$
F_{1}(t, x)=\left(\frac{1}{2} T-t\right) \ln ^{\frac{7}{4}}\left(1+|x|^{2}\right)+\left(\frac{2}{3} T-t\right) \ln \left(1+|x|^{2}\right),
$$

then

$$
\left|\nabla F_{1}(t, x)\right| \leq \frac{7}{4}\left|\frac{1}{2} T-t\right| \ln ^{\frac{3}{4}}\left(1+|x|^{2}\right)+\left|\frac{2}{3} T-t\right|
$$


by some simple computations, we can show that the conditions $\left(\mathrm{H}_{1}\right)$ and $\left(\mathrm{H}_{2}\right)$ of Theorem 3.1 hold with control function $h(|x|)=\ln ^{\frac{3}{4}}\left(1+|x|^{2}\right)$. Let $F_{2}(x)=-\frac{4 d}{5}|x|^{\frac{5}{4}}$, where $d>0$, then the conditions $\left(\mathrm{H}_{3}\right)$ and $\left(\mathrm{H}_{5}\right)$ of Theorem 3.1 holds.

Remark 3.5 In the case $M(s) \equiv 1$ and $p(t) \equiv 2$, we note that in [6], the Ahmad-Lazer-Paultype coercive condition was assumed:

$$
\lim _{|x| \rightarrow \infty} \frac{1}{|x|^{2 \alpha}} \int_{0}^{T} F(t, x) d t=+\infty \quad(\text { or }-\infty)
$$

In [2], Wang and Zhang use the following condition instead of condition (3.1):

$$
\lim _{|x| \rightarrow \infty} \frac{1}{h^{2}(|x|)} \int_{0}^{T} F(t, x) d t=+\infty \quad(\text { or }-\infty)
$$

In this paper, we generalized (3.2) to the condition $\left(\mathrm{H}_{4}\right)$ or $\left(\mathrm{H}_{6}\right)$, and it is easy to see that

$$
\liminf _{|x| \rightarrow \infty} \frac{1}{h^{q^{+}}(|x|)} \int_{0}^{T} F(t, x) d t \quad \text { or } \quad \limsup _{|x| \rightarrow \infty} \frac{1}{h^{q^{+}}(|x|)} \int_{0}^{T} F(t, x) d t
$$

has appropriate lower and upper bounds.

Remark 3.6 Theorem 3.1 and Theorem 3.2 extend some classical results for second order Hamiltonian systems to the nonlocal and variable exponent space setting:

(a) When $M(s) \equiv 1, p(t) \equiv 2$ and $F_{2}=0$, Theorems 1.1 and 1.2 in [2] are direct corollaries of our Theorems 3.1 and 3.2, respectively.

(b) When $M(s) \equiv 1, p(t) \equiv 2$, Theorems 1.1 and 1.2 in [3], Theorems 1 and 2 in [4], Theorems 1.1 and 1.2 in [5] and Theorems 1 and 2 in [6] are special cases of our Theorems 3.1 and 3.2 with $F_{2}=0$ or $h(t)=t^{\alpha}, \alpha \in[0,1), t \in[0,+\infty)$, respectively.

Finally, we shall obtain the existence of multiple periodic solutions for systems (1.1). For our result, we further assume that the following conditions hold:

$\left(\mathrm{M}_{2}\right)$ There exists a constant $\eta \geq 1$ such that

$$
\eta \widehat{M}(s)=\eta \int_{0}^{s} M(\sigma) d \sigma \geq M(s) s
$$

for all $s \in[0,+\infty)$.

$\left(\mathrm{H}_{7}\right) \quad \lim \sup _{|x| \rightarrow 0} \frac{|F(t, x)|}{|x| p^{+}} \leq \frac{m_{0}}{2 p^{+} T d_{0}^{p^{+}}}$, uniformly for a.e. $t \in[0, T]$, where $d_{0}$ is a positive constant which satisfies (2.3).

$\left(\mathrm{H}_{8}\right)$ There exists $\delta>0$ such that $F(t, x) \leq 0$ for $x \in \mathbb{R}^{N}$ with $|x| \leq \delta$ and a.e. $t \in[0, T]$.

$\left(\mathrm{H}_{9}\right) \quad F(t, x)$ is even in $x$ and $F(t, 0)=0$.

$\left(\mathrm{H}_{10}\right) \lim _{|x| \rightarrow 0} \frac{F(t, x)}{\left.|x|\right|^{p p^{-}}}=-\infty$.

Theorem 3.7 Suppose that assumption (A), conditions $\left(\mathrm{M}_{0}\right),\left(\mathrm{M}_{1}\right),\left(\mathrm{H}_{1}\right)-\left(\mathrm{H}_{4}\right),\left(\mathrm{H}_{7}\right)$, and $\left(\mathrm{H}_{8}\right)$ hold, then problem (1.1) has at least two distinct T-periodic solutions.

Theorem 3.8 Suppose that assumption (A), conditions $\left(\mathrm{M}_{0}\right)-\left(\mathrm{M}_{2}\right),\left(\mathrm{H}_{1}\right)-\left(\mathrm{H}_{3}\right),\left(\mathrm{H}_{5}\right),\left(\mathrm{H}_{6}\right)$, $\left(\mathrm{H}_{9}\right)$, and $\left(\mathrm{H}_{10}\right)$ hold, then problem (1.1) has infinitely many nontrivial T-periodic solutions. 


\section{Proofs of Theorem 3.1 and Theorem 3.2}

The Euler-Lagrange functional associated to problem (1.1) is given by

$$
\Phi(u)=\widehat{M}\left(\int_{0}^{T} \frac{|\dot{u}(t)|^{p(t)}}{p(t)} d t\right)+\int_{0}^{T} F(t, u(t)) d t
$$

for all $u \in W_{T}^{1, p(t)}$, where we recall that

$$
\widehat{M}(s)=\int_{0}^{s} M(\sigma) d \sigma .
$$

From the assumptions on $M$ and $F$, it is standard to check that $\Phi$ is continuously differentiable and weakly semicontinuous on $W_{T}^{1, p(t)}$. Moreover, we have

$$
\begin{aligned}
\left\langle\Phi^{\prime}(u), v\right\rangle= & M\left(\int_{0}^{T} \frac{|\dot{u}(t)|^{p(t)}}{p(t)} d t\right) \int_{0}^{T}\left(|\dot{u}(t)|^{p(t)-2} \dot{u}(t), \dot{v}(t)\right) d t \\
& +\int_{0}^{T}(\nabla F(t, u(t)), v(t)) d t
\end{aligned}
$$

for all $u, v \in W_{T}^{1, p(t)}$.

A critical point of $\Phi$ is a solution $\omega$ of $\left\langle\Phi^{\prime}(\omega), v\right\rangle=0$ for all $v \in W_{T}^{1, p(t)}$. Let $\left\langle\Phi^{\prime}(\omega), v\right\rangle=0$ for all $\omega \in W_{T}^{1, p(t)}$, if and only if

$$
M\left(\int_{0}^{T} \frac{|\dot{\omega}(t)|^{p(t)}}{p(t)} d t\right) \int_{0}^{T}\left(|\dot{\omega}(t)|^{p(t)-2} \dot{u}(t), \dot{v}(t)\right) d t+\int_{0}^{T}(\nabla F(t, \omega(t)), v(t)) d t=0 .
$$

Since $\omega \in W_{T}^{1, p(t)}$, the $T$-periodic solutions of problem (1.1) correspond to the critical points of the functional $\Phi$ in $W_{T}^{1, p(1)}$.

In the sequel, we denote $C_{i}(i=0,1,2,3, \ldots)$, which are various positive constants. Now we begin to prove our main results.

For $u \in W_{T}^{1, p(t)}$, let $\bar{u}=\frac{1}{T} \int_{0}^{T} u(t) d t$ and $\tilde{u}(t)=u(t)-\bar{u}$.

Proof of Theorem 3.1 From $\left(\mathrm{M}_{0}\right)$, we obtain

$$
\begin{aligned}
\widehat{M}\left(\int_{0}^{T} \frac{|\dot{u}(t)|^{p(t)}}{p(t)} d t\right) & =\int_{0}^{\int_{0}^{T} \frac{|\dot{u}(t)|^{p(t)}}{p(t)} d t} M(s) d s \\
& \geq m_{0} \int_{0}^{T} \frac{|\dot{u}(t)|^{p(t)}}{p(t)} d t \\
& \geq \frac{m_{0}}{p^{+}} \int_{0}^{T}|\dot{u}(t)|^{p(t)} d t .
\end{aligned}
$$

By Young's inequality, we obtain

$$
\begin{aligned}
& 2 K_{0} C_{0} \int_{0}^{T} f(t) d t h(|\bar{u}|)\left(\int_{0}^{T}|\dot{u}(t)|^{p(t)} d t\right)^{\frac{1}{p^{-}}} \\
& \quad=2 K_{0} C_{1} \int_{0}^{T} f(t) d t h(|\bar{u}|)\left(\frac{\theta p^{+}}{m_{0} p^{-}}\right)^{\frac{1}{p^{-}}}\left(\frac{m_{0} p^{-}}{\theta p^{+}}\right)^{\frac{1}{p^{-}}}\left(\int_{0}^{T}|\dot{u}(t)|^{p(t)} d t\right)^{\frac{1}{p^{-}}}
\end{aligned}
$$




$$
\begin{aligned}
\leq & \frac{1}{q^{+}}\left(\frac{\theta p^{+}}{m_{0} p^{-}}\right)^{q^{+}}\left(2 K_{0} C_{0} \int_{0}^{T} f(t) d t\right)^{q^{+}} h^{q^{+}}(|\bar{u}|) \\
& +\frac{m_{0}}{\theta p^{+}} \int_{0}^{T}|\dot{u}(t)|^{p(t)} d t
\end{aligned}
$$

where $\frac{1}{p^{-}}+\frac{1}{q^{+}}=1$.

It follows from $\left(\mathrm{H}_{1}\right),\left(\mathrm{H}_{2}\right),(2.1)$, and (4.2) that

$$
\begin{aligned}
& \left|\int_{0}^{T}\left[F_{1}(t, u(t))-F_{1}(t, \bar{u})\right] d t\right| \\
& =\left|\int_{0}^{T} \int_{0}^{1}\left(\nabla F_{1}(t, \bar{u}+s \tilde{u}(t)), \tilde{u}(t)\right) d s d t\right| \\
& \leq \int_{0}^{T} \int_{0}^{1} f(t) h(|\bar{u}+s \tilde{u}(t)|)|\tilde{u}(t)| d s d t+\int_{0}^{T} \int_{0}^{1} g(t)|\tilde{u}(t)| d s d t \\
& \leq \int_{0}^{T} \int_{0}^{1} f(t) K_{0}[h(|\bar{u}|)+h(|\tilde{u}(t)|)]|\tilde{u}(t)| d s d t \\
& +\int_{0}^{T} \int_{0}^{1} g(t)|\tilde{u}(t)| d s d t \\
& \leq K_{0} \int_{0}^{T} \int_{0}^{1} f(t)\left[h(|\bar{u}|)+K_{1}|\tilde{u}(t)|^{\alpha}+K_{2}\right]|\tilde{u}(t)| d s d t \\
& +\int_{0}^{T} \int_{0}^{1} g(t)|\tilde{u}(t)| d s d t \\
& \leq h(|\bar{u}|)\|\tilde{u}\|_{\infty} K_{0} \int_{0}^{T} f(t) d t+\|\tilde{u}\|_{\infty}^{\alpha+1} K_{0} K_{1} \int_{0}^{T} f(t) d t \\
& +\|\tilde{u}\|_{\infty}\left[K_{0} K_{2} \int_{0}^{T} f(t) d t+\int_{0}^{T} g(t) d t\right] \\
& \leq h(|\bar{u}|)\left[2 C_{0}\left(\int_{0}^{T}|\dot{u}(t)|^{p(t)} d t\right)^{\frac{1}{p^{-}}}+C_{1}\right] K_{0} \int_{0}^{T} f(t) d t \\
& +\left[2 C_{0}\left(\int_{0}^{T}|\dot{u}(t)|^{p(t)} d t\right)^{\frac{1}{p^{-}}}+C_{1}\right]^{\alpha+1} K_{0} K_{1} \int_{0}^{T} f(t) d t \\
& +\left[2 C_{0}\left(\int_{0}^{T}|\dot{u}(t)|^{p(t)} d t\right)^{\frac{1}{p^{-}}}+C_{1}\right]\left[K_{0} K_{2} \int_{0}^{T} f(t) d t+\int_{0}^{T} g(t) d t\right] \\
& \leq 2 K_{0} C_{0} \int_{0}^{T} f(t) d t h(|\bar{u}|)\left(\int_{0}^{T}|\dot{u}(t)|^{p(t)} d t\right)^{\frac{1}{p^{-}}} \\
& +C_{3}\left(\int_{0}^{T}|\dot{u}(t)|^{p(t)} d t\right)^{\frac{\alpha+1}{p-1}}+C_{4}\left(\int_{0}^{T}|\dot{u}(t)|^{p(t)} d t\right)^{\frac{1}{p^{-}}} \\
& +C_{5} h(|\bar{u}|)+C_{6} \\
& \leq \frac{m_{0}}{\theta p^{+}} \int_{0}^{T}|\dot{u}(t)|^{p(t)} d t+C_{3}\left(\int_{0}^{T}|\dot{u}(t)|^{p(t)} d t\right)^{\frac{\alpha+1}{p}}+C_{4}\left(\int_{0}^{T}|\dot{u}(t)|^{p(t)} d t\right)^{\frac{1}{p^{-}}} \\
& +\frac{1}{q^{+}}\left(2 K_{0} C_{0} \int_{0}^{T} f(t) d t\right)^{q^{+}}\left(\frac{\theta p^{+}}{m_{0} p^{-}}\right)^{\frac{q^{+}}{p}} h^{q^{+}}(|\bar{u}|)+C_{5} h(|\bar{u}|)+C_{6} .
\end{aligned}
$$


Similarly, by $\left(\mathrm{H}_{3}\right)$ and (2.1), for any $u \in W_{T}^{1, p(t)}$, we get

$$
\begin{aligned}
\int_{0}^{T}\left[F_{2}(u(t))-F_{2}(\bar{u})\right] d t & =\int_{0}^{T} \int_{0}^{1} \frac{1}{s}\left(\nabla F_{2}(\bar{u}+s \tilde{u}(t))-\nabla F_{2}(\bar{u}), s \tilde{u}(t)\right) d s d t \\
& \geq-\int_{0}^{T} \int_{0}^{1} r s^{\gamma-1}|\tilde{u}(t)|^{\gamma} d s d t \\
& \geq-\frac{r T}{\gamma}\|\tilde{u}\|_{\infty}^{\gamma} \\
& \geq-\frac{r T}{\gamma}\left[2 C_{0}\left(\int_{0}^{T}|\dot{u}(t)|^{p(t)} d t\right)^{\frac{1}{p^{-}}}+C_{1}\right]^{\gamma} \\
& \geq-C_{7}\left(\int_{0}^{T}|\dot{u}(t)|^{p(t)} d t\right)^{\frac{\gamma}{p^{-}}}-C_{8} .
\end{aligned}
$$

Taking into account (4.1), (4.3), and (4.4) we obtain

$$
\begin{aligned}
\Phi(u)= & \widehat{M}\left(\int_{0}^{T} \frac{|\dot{u}(t)|^{p(t)}}{p(t)} d t\right)+\int_{0}^{T} F(t, u(t)) d t \\
\geq & \frac{m_{0}}{p^{+}} \int_{0}^{T}|\dot{u}(t)|^{p(t)} d t+\int_{0}^{T}\left[F_{1}(t, u(t))-F_{1}(t, \bar{u})\right] d t \\
& +\int_{0}^{T}\left[F_{2}(u(t))-F_{2}(\bar{u})\right] d t+\int_{0}^{T} F(t, \bar{u}) d t \\
\geq & \frac{m_{0}}{p^{+}}\left(1-\frac{1}{\theta}\right) \int_{0}^{T}|\dot{u}(t)|^{p(t)} d t-C_{3}\left(\int_{0}^{T}|\dot{u}(t)|^{p(t)} d t\right)^{\frac{\alpha+1}{p^{-}}} \\
& -C_{7}\left(\int_{0}^{T}|\dot{u}(t)|^{p(t)} d t\right)^{\frac{\gamma}{p^{-}}}-C_{4}\left(\int_{0}^{T}|\dot{u}(t)|^{p(t)} d t\right)^{\frac{1}{p^{-}}} \\
& +\left[\frac{1}{h^{q^{+}}(|\bar{u}|)} \int_{0}^{T} F(t, \bar{u}) d t-\frac{1}{q^{+}}\left(2 K_{0} C_{0} \int_{0}^{T} f(t) d t\right)^{q^{+}}\left(\frac{\theta p^{+}}{m_{0} p^{-}}\right)^{q^{-}}\right] h^{q^{+}}(|\bar{u}|) \\
& -C_{5} h(|\bar{u}|)-C_{9} .
\end{aligned}
$$

It follows from Lemma 2.4 that

$$
\|u\| \rightarrow \infty \Rightarrow \int_{0}^{T}|\dot{u}(t)|^{p(t)} d t+|\bar{u}| \rightarrow+\infty .
$$

Combining with the above inequality, condition $\left(H_{1}\right)$ and $\theta>1$ imply that

$$
\Phi(u) \rightarrow+\infty \quad \text { as }\|u\| \rightarrow \infty
$$

Note that $W_{T}^{1, p(t)}$ is reflexive Banach space, and the functional $\Phi$ is weakly lower semicontinuous, applying the least action principle (see [1], Theorem 1.1 and Corollary 1.1), $\Phi$ has a minimum point on $W_{T}^{1, p(t)}$, which is a critical point of $\Phi$. The proof is complete.

Proof of Theorem 3.2 Step 1: We prove that $\Phi$ satisfies the (PS) condition. Suppose that $\left\{u_{n}\right\} \subset W_{T}^{1, p(t)}$ is a (PS) sequence of $\Phi$, that is, $\Phi^{\prime}\left(u_{n}\right) \rightarrow 0$, as $n \rightarrow \infty$, and $\left\{\Phi\left(u_{n}\right)\right\}$ is 
bounded. In a way similar to the proof of Theorem 3.1, we have

$$
\begin{aligned}
& \left|\int_{0}^{T}\left(\nabla F_{1}\left(t, u_{n}(t)\right), \tilde{u}_{n}(t)\right) d t\right| \\
& \leq \frac{m_{0}}{\theta p^{+}} \int_{0}^{T}\left|\dot{u}_{n}(t)\right|^{p(t)} d t+C_{3}\left(\int_{0}^{T}\left|\dot{u}_{n}(t)\right|^{p(t)} d t\right)^{\frac{\alpha+1}{p^{-}}} \\
& \quad+C_{4}\left(\int_{0}^{T}\left|\dot{u}_{n}(t)\right|^{p(t)} d t\right)^{\frac{1}{p^{-}}}+C_{5} h\left(\left|\bar{u}_{n}\right|\right)+C_{6} \\
& \quad+\frac{1}{q^{+}}\left(2 K_{0} C_{0} \int_{0}^{T} f(t) d t\right)^{q^{+}}\left(\frac{\theta p^{+}}{m_{0} p^{-}}\right)^{\frac{q^{+}}{p^{-}}} h^{q^{+}}\left(\left|\bar{u}_{n}\right|\right)
\end{aligned}
$$

and

$$
\int_{0}^{T}\left(\nabla F_{2}\left(u_{n}(t)\right), \tilde{u}_{n}(t)\right) d t \geq-C_{7}\left(\int_{0}^{T}\left|\dot{u}_{n}(t)\right|^{p(t)} d t\right)^{\frac{\gamma}{p^{-}}}-C_{8} .
$$

It follows from $\left(\mathrm{M}_{0}\right)$, (4.5), and (4.6) that

$$
\begin{aligned}
\left\|\tilde{u}_{n}\right\| \geq & \left\langle\Phi^{\prime}\left(u_{n}\right), \tilde{u}_{n}\right\rangle \\
= & M\left(\int_{0}^{T} \frac{\left|\dot{u}_{n}(t)\right|^{p(t)}}{p(t)} d t\right) \int_{0}^{T}\left|\dot{u}_{n}(t)\right|^{p(t)} d t+\int_{0}^{T}\left(\nabla F\left(t, u_{n}(t)\right), \tilde{u}_{n}(t)\right) d t \\
\geq & m_{0} \int_{0}^{T}\left|\dot{u}_{n}(t)\right|^{p(t)} d t+\int_{0}^{T}\left(\nabla F_{1}\left(t, u_{n}(t)\right), \tilde{u}_{n}(t)\right) d t \\
& +\int_{0}^{T}\left(\nabla F_{2}\left(u_{n}(t)\right), \tilde{u}_{n}(t)\right) d t \\
\geq & m_{0}\left(1-\frac{1}{\theta p^{+}}\right) \int_{0}^{T}\left|\dot{u}_{n}(t)\right|^{p(t)} d t-C_{3}\left(\int_{0}^{T}\left|\dot{u}_{n}(t)\right|^{p(t)} d t\right)^{\frac{\alpha+1}{p-}} \\
& -C_{4}\left(\int_{0}^{T}\left|\dot{u}_{n}(t)\right|^{p(t)} d t\right)^{\frac{1}{p^{-}}}-C_{7}\left(\int_{0}^{T}\left|\dot{u}_{n}(t)\right|^{p(t)} d t\right)^{\frac{\gamma}{p^{-}}} \\
& -\frac{1}{q^{+}}\left[2 K_{0} C_{0} \int_{0}^{T} f(t) d t\left(\frac{\theta p^{+}}{m_{0} p^{-}}\right)^{\frac{1}{p^{-}}}\right]^{q^{+}} h^{q^{+}}\left(\left|\bar{u}_{n}\right|\right) \\
& -C_{5} h\left(\left|\bar{u}_{n}\right|\right)-C_{10}
\end{aligned}
$$

for large $n$. By (2.2) we have

$$
\left\|\tilde{u}_{n}\right\| \leq C_{2}\left[\left(\int_{0}^{T}\left|\dot{u}_{n}(t)\right|^{p(t)} d t\right)^{\frac{1}{p^{-}}}+1\right] .
$$

Combining (4.7) and (4.8), we have

$$
\begin{aligned}
& \frac{1}{q^{+}}\left[2 K_{0} C_{0} \int_{0}^{T} f(t) d t\left(\frac{\theta p^{+}}{m_{0} p^{-}}\right)^{\frac{1}{p^{-}}}\right]^{q^{+}} h^{q^{+}}\left(\left|\bar{u}_{n}\right|\right)+C_{5} h\left(\left|\bar{u}_{n}\right|\right) \\
& \quad \geq m_{0}\left(1-\frac{1}{\theta p^{+}}\right) \int_{0}^{T}\left|\dot{u}_{n}(t)\right|^{p(t)} d t-C_{3}\left(\int_{0}^{T}\left|\dot{u}_{n}(t)\right|^{p(t)} d t\right)^{\frac{\alpha+1}{p^{-}}}
\end{aligned}
$$




$$
\begin{aligned}
& -C_{7}\left(\int_{0}^{T}\left|\dot{u}_{n}(t)\right|^{p(t)} d t\right)^{\frac{\gamma}{p^{-}}}-C_{10}\left(\int_{0}^{T}\left|\dot{u}_{n}(t)\right|^{p(t)} d t\right)^{\frac{1}{p^{-}}}-C_{11} \\
\geq & \frac{m_{0}}{2}\left(1-\frac{1}{\theta p^{+}}\right) \int_{0}^{T}\left|\dot{u}_{n}(t)\right|^{p(t)} d t-C_{12},
\end{aligned}
$$

where $\theta>1, \gamma \in\left[0, p^{-}\right), \alpha \in\left[0, p^{-}-1\right)$, and

$$
C_{12}=-\min _{s \in[0,+\infty)}\left\{\frac{m_{0}}{2}\left(1-\frac{1}{\theta p^{+}}\right) s^{p^{-}}-C_{3} s^{\alpha+1}-C_{7} s^{\gamma}-C_{10} s-C_{11}\right\}>0 .
$$

Then equation (4.9) implies that

$$
\begin{aligned}
\int_{0}^{T}\left|\dot{u}_{n}(t)\right|^{p(t)} d t \leq & \frac{1}{\frac{m_{0}}{2}\left(1-\frac{1}{\theta p^{+}}\right)} \frac{1}{q^{+}}\left(2 K_{0} C_{0} \int_{0}^{T} f(t) d t\right)^{q^{+}}\left(\frac{\theta p^{+}}{m_{0} p^{-}}\right)^{\frac{q^{+}}{p^{-}}} h^{q^{+}}\left(\left|\bar{u}_{n}\right|\right) \\
& +C_{13} h\left(\left|\bar{u}_{n}\right|\right)+C_{14} .
\end{aligned}
$$

From $\left(\mathrm{M}_{1}\right)$, we have

$$
\begin{aligned}
\frac{1}{p^{-}}\left\|\tilde{u}_{n}\right\| \geq & \frac{1}{p^{-}}\left\langle\Phi^{\prime}\left(u_{n}\right), \tilde{u}_{n}\right\rangle \\
= & \frac{1}{p^{-}} M\left(\int_{0}^{T} \frac{\left|\dot{u}_{n}(t)\right|^{p(t)}}{p(t)} d t\right) \int_{0}^{T}\left|\dot{u}_{n}(t)\right|^{p(t)} d t \\
& +\frac{1}{p^{-}} \int_{0}^{T}\left(\nabla F\left(t, u_{n}(t)\right), \tilde{u}_{n}(t)\right) d t \\
\geq & M\left(\int_{0}^{T} \frac{\left|\dot{u}_{n}(t)\right|^{p(t)}}{p(t)} d t\right) \int_{0}^{T} \frac{\left|\dot{u}_{n}(t)\right|^{p(t)}}{p(t)} d t \\
& +\frac{1}{p^{-}} \int_{0}^{T}\left(\nabla F\left(t, u_{n}(t)\right), \tilde{u}_{n}(t)\right) d t \\
\geq & \widehat{M}\left(\int_{0}^{T} \frac{\left|\dot{u}_{n}(t)\right|^{p(t)}}{p(t)} d t\right)+\frac{1}{p^{-}} \int_{0}^{T}\left(\nabla F_{1}\left(t, u_{n}(t)\right), \tilde{u}_{n}(t)\right) d t \\
& +\frac{1}{p^{-}} \int_{0}^{T}\left(\nabla F_{2}\left(u_{n}(t)\right), \tilde{u}_{n}(t)\right) d t .
\end{aligned}
$$

Combining (4.5), (4.6) (4.8), (4.10), and (4.11), we have

$$
\begin{aligned}
\widehat{M}\left(\int_{0}^{T} \frac{\left|\dot{u}_{n}(t)\right|^{p(t)}}{p(t)} d t\right) \leq & \frac{1}{p^{-}}\left\|\tilde{u}_{n}\right\|-\frac{1}{p^{-}} \int_{0}^{T}\left(\nabla F_{2}\left(u_{n}(t)\right), \tilde{u}_{n}(t)\right) d t \\
& -\frac{1}{p^{-}} \int_{0}^{T}\left(\nabla F_{1}\left(t, u_{n}(t)\right), \tilde{u}_{n}(t)\right) d t \\
\leq & \frac{C_{2}}{p^{-}}\left[\left(\int_{0}^{T}\left|\dot{u}_{n}(t)\right|^{p(t)} d t\right)^{\frac{1}{p^{-}}}+1\right] \\
& -\frac{1}{p^{-}} \int_{0}^{T}\left(\nabla F_{2}\left(u_{n}(t)\right), \tilde{u}_{n}(t)\right) d t \\
& -\frac{1}{p^{-}} \int_{0}^{T}\left(\nabla F_{1}\left(t, u_{n}(t)\right), \tilde{u}_{n}(t)\right) d t
\end{aligned}
$$


Chang Boundary Value Problems ( 2016) 2016:211

Page 13 of 20

$$
\begin{aligned}
\leq & \frac{1}{p^{-}} \frac{m_{0}}{\theta p^{+}} \int_{0}^{T}\left|\dot{u}_{n}(t)\right|^{p(t)} d t+C_{3}\left(\int_{0}^{T}\left|\dot{u}_{n}(t)\right|^{p(t)} d t\right)^{\frac{\alpha+1}{p^{-}}} \\
& +C_{7}\left(\int_{0}^{T}\left|\dot{u}_{n}(t)\right|^{p(t)} d t\right)^{\frac{\gamma}{p^{-}}}+C_{15}\left(\int_{0}^{T}|\dot{u}(t)|^{p(t)} d t\right)^{\frac{1}{p^{-}}} \\
& +\frac{1}{p^{-}} \frac{1}{q^{+}}\left(2 K_{0} C_{0} \int_{0}^{T} f(t) d t\right)^{q^{+}}\left(\frac{\theta p^{+}}{m_{0} p^{-}}\right)^{\frac{q^{+}}{p^{-}}} h^{q^{+}}\left(\left|\bar{u}_{n}\right|\right) \\
& +C_{16} h\left(\left|\bar{u}_{n}\right|\right)+C_{17} \\
\leq & \frac{\theta p^{+}+1}{p^{-} q^{+}\left(\theta p^{+}-1\right)}\left(2 K_{0} C_{0} \int_{0}^{T} f(t) d t\right)^{q^{+}}\left(\frac{2 \eta p^{+}}{m_{0} p^{-}}\right)^{\frac{q^{+}}{p^{-}}} h^{q^{+}}\left(\left|\bar{u}_{n}\right|\right) \\
& +C_{18} h^{\frac{(\alpha+1) q^{+}}{p^{-}}}\left(\left|\bar{u}_{n}\right|\right)+C_{19} h^{\frac{q^{+}}{p^{-}}}\left(\left|\bar{u}_{n}\right|\right)+C_{20} h^{\frac{\gamma q^{+}}{p^{-}}}\left(\left|\bar{u}_{n}\right|\right) \\
& +C_{21} h\left(\left|\bar{u}_{n}\right|\right)+C_{22} h^{\frac{\alpha+1}{p^{-}}}\left(\left|\bar{u}_{n}\right|\right)+C_{23} h^{\frac{\gamma}{p^{-}}}\left(\left|\bar{u}_{n}\right|\right) \\
& +C_{24} h^{\frac{1}{p^{-}}}\left(\left|\bar{u}_{n}\right|\right)+C_{25} .
\end{aligned}
$$

By $\left(\mathrm{H}_{5}\right)$ and (2.1), similar to the proof of (4.4), one has

$$
\int_{0}^{T}\left[F_{2}(u(t))-F_{2}(\bar{u})\right] d t \leq C_{26}\left(\int_{0}^{T}|\dot{u}(t)|^{p(t)} d t\right)^{\frac{\beta}{p^{-}}}+C_{27} .
$$

In view of (4.3), (4.10), and (4.13), we obtain

$$
\begin{aligned}
\int_{0}^{T}[ & F(t, u(t))-F(t, \bar{u})] d t \\
= & \int_{0}^{T}\left[F_{1}(t, u(t))-F_{1}(t, \bar{u})\right] d t+\int_{0}^{T}\left[F_{2}(u(t))-F_{2}(\bar{u})\right] d t \\
\leq & \frac{1}{q^{+}} \frac{\theta p^{+}+1}{\theta p^{+}-1}\left[2 K_{0} C_{0} \int_{0}^{T} f(t) d t\left(\frac{\theta p^{+}}{m_{0} p^{-}}\right)^{\frac{1}{p^{-}}}\right]^{q^{+}} h^{q^{+}}\left(\left|\bar{u}_{n}\right|\right) \\
& +C_{28} h^{\frac{(\alpha+1) q^{+}}{p^{-}}}\left(\left|\bar{u}_{n}\right|\right)+C_{29} h^{\frac{q^{+}}{p^{-}}}\left(\left|\bar{u}_{n}\right|\right)+C_{30} h^{\frac{\beta q^{+}}{p^{-}}}\left(\left|\bar{u}_{n}\right|\right) \\
& +C_{31} h^{\frac{\alpha+1}{p^{-}}}\left(\left|\bar{u}_{n}\right|\right)+C_{32} h\left(\left|\bar{u}_{n}\right|\right)+C_{33} h^{\frac{\beta}{p^{-}}}\left(\left|\bar{u}_{n}\right|\right) \\
& +C_{34} h^{\frac{1}{p^{-}}}\left(\left|\bar{u}_{n}\right|\right)+C_{35} .
\end{aligned}
$$

Hence, combining (4.12) and (4.14), a direct calculation yields

$$
\begin{aligned}
\Phi\left(u_{n}\right)= & \widehat{M}\left(\int_{0}^{T} \frac{\left|\dot{u}_{n}(t)\right|^{p(t)}}{p(t)} d t\right)+\int_{0}^{T}\left[F\left(t, u_{n}(t)\right)-F\left(t, \bar{u}_{n}\right)\right] d t \\
& +\int_{0}^{T} F\left(t, \bar{u}_{n}\right) d t \\
\leq & {\left[\frac{\int_{0}^{T} F\left(t, \bar{u}_{n}\right) d t}{h^{q^{+}}\left(\left|\bar{u}_{n}\right|\right)}+K\right] h^{q^{+}}\left(\left|\bar{u}_{n}\right|\right)+C_{36} h^{\frac{(\alpha+1) q^{+}}{p^{-}}}\left(\left|\bar{u}_{n}\right|\right)+C_{37} h^{\frac{q^{+}}{p^{-}}}\left(\left|\bar{u}_{n}\right|\right) } \\
& +C_{38} h^{\frac{\alpha+1}{p^{-}}}\left(\left|\bar{u}_{n}\right|\right)+C_{39} h\left(\left|\bar{u}_{n}\right|\right)+C_{40} h^{\frac{1}{p^{-}}}\left(\left|\bar{u}_{n}\right|\right)+C_{20} h^{\frac{\gamma q^{+}}{p^{-}}}\left(\left|\bar{u}_{n}\right|\right) \\
& +C_{23} h^{\frac{\gamma}{p^{-}}}\left(\left|\bar{u}_{n}\right|\right)+C_{30} h^{\frac{\beta q^{+}}{p^{-}}}\left(\left|\bar{u}_{n}\right|\right)+C_{33} h^{\frac{\beta}{p^{-}}}\left(\left|\bar{u}_{n}\right|\right)+C_{41},
\end{aligned}
$$


where $K$ is defined as $\left(\mathrm{H}_{6}\right)$. We claim that the sequence $\left\{u_{n}\right\}$ is bounded in $W_{T}^{1, p(t)}$. Suppose that is not the case, passing to a subsequence if necessary, we may assume that

$$
\left\|u_{n}\right\| \rightarrow+\infty \text { as } n \rightarrow \infty
$$

Applying Lemma 2.4, we obtain

$$
\left\|u_{n}\right\| \rightarrow \infty \Rightarrow \int_{0}^{T}\left|\dot{u}_{n}(t)\right|^{p(t)} d t+\left|\bar{u}_{n}\right| \rightarrow+\infty \quad \text { as } n \rightarrow \infty .
$$

If $\left|\bar{u}_{n}\right| \rightarrow+\infty$ as $n \rightarrow \infty$. By $\left(\mathrm{H}_{1}\right)$, we obtain $h\left(\left|\bar{u}_{n}\right|\right) \rightarrow+\infty$ as $n \rightarrow \infty$. Exploiting the facts $\gamma \in\left[0, p^{-}\right), \beta \in\left[0, p^{-}\right), \alpha \in\left[0, p^{-}-1\right), p^{-}>1$ and $q^{+}>1$, using condition $\left(\mathrm{H}_{6}\right)$ and (4.15), we can see that $\Phi\left(u_{n}\right) \rightarrow-\infty$ as $n \rightarrow \infty$. If $\int_{0}^{T}\left|\dot{u}_{n}(t)\right|^{p(t)} d t \rightarrow+\infty$ as $n \rightarrow \infty$. By (4.10), we obtain $h\left(\left|\bar{u}_{n}\right|\right) \rightarrow+\infty$ as $n \rightarrow \infty$. From $\left(\mathrm{H}_{6}\right)$ and (4.15), we deduce that $\Phi\left(u_{n}\right) \rightarrow-\infty$ as $n \rightarrow \infty$, which is contrary to the boundedness of $\left\{\Phi\left(u_{n}\right)\right\}$. Thus, $\left\{u_{n}\right\}$ is bounded in $W_{T}^{1, p(t)}$.

Next, we show that the energy functional $\Phi$ satisfies (PS) condition. The sequence $\left\{u_{n}\right\} \subset$ $W_{T}^{1, p(t)}$ has a subsequence, also denoted by $\left\{u_{n}\right\}$, such that

$$
u_{n} \rightarrow u \quad \text { weakly in } W_{T}^{1, p(t)} \text { and } \quad u_{n} \rightarrow u \quad \text { strongly in } C\left([0, T] ; \mathbb{R}^{N}\right) .
$$

Consequently, we get

$$
\begin{aligned}
\left|\int_{0}^{T}\left(\nabla F\left(t, u_{n}(t)\right), u_{n}(t)-u(t)\right) d t\right| & \leq T\left\|u_{n}-u\right\|_{\infty} \max _{0 \leq t \leq T}\left|\nabla F\left(t, u_{n}(t)\right)\right| \\
& \rightarrow 0 \quad \text { as } n \rightarrow \infty .
\end{aligned}
$$

Note that $\left\{u_{n}\right\} \subset W_{T}^{1, p(t)}$ is a (PS) sequence of $\Phi$ and we have $\left\langle\Phi^{\prime}\left(u_{n}\right), u_{n}-u\right\rangle \rightarrow 0$ as $n \rightarrow$ $\infty$, and we have

$$
M\left(\int_{0}^{T}\left|\dot{u}_{n}(t)\right|^{p(t)} d t\right) \int_{0}^{T}\left(\left|\dot{u}_{n}(t)\right|^{p(t)-2} \dot{u}_{n}(t), \dot{u}_{n}(t)-\dot{u}(t)\right) d t \rightarrow 0 \quad \text { as } n \rightarrow \infty .
$$

Using $\left(\mathrm{M}_{0}\right)$, we have

$$
\left(J^{\prime}\left(u_{n}\right), u_{n}-u\right)=\int_{0}^{T}\left(\left|\dot{u}_{n}(t)\right|^{p(t)-2} \dot{u}_{n}(t), \dot{u}_{n}(t)-\dot{u}(t)\right) d t \rightarrow 0 \quad \text { as } n \rightarrow \infty .
$$

Furthermore, since $J^{\prime}(u)$ is a bounded linear function, we get $\left(J^{\prime}(u), u_{n}-u\right) \rightarrow 0$ as $n \rightarrow \infty$. Thus $\lim _{n \rightarrow \infty}\left(J^{\prime}\left(u_{n}\right)-J^{\prime}(u), u_{n}-u\right)=0$. By Lemma 2.6, we see that $\left\{u_{n}\right\}$ admits a convergent sequence. Thus functional $\Phi$ satisfies condition (PS).

Step 2: We prove that $\Phi$ satisfies the other conditions of the saddle point theorem.

On one hand, for $u \in \widetilde{W}_{T}^{1, p(t)}$, similar to the proof of (4.3), we have

$$
\begin{aligned}
& \left|\int_{0}^{T}\left[F_{1}(t, u(t))-F_{1}(t, 0)\right] d t\right| \\
& \quad=\left|\int_{0}^{T} \int_{0}^{1}\left(\nabla F_{1}(t, s u(t)), u(t)\right) d s d t\right|
\end{aligned}
$$




$$
\begin{aligned}
& \leq \int_{0}^{T} f(t) h(|u(t)|)|u(t)| d t+\int_{0}^{T} g(t)|u(t)| d t \\
& \leq \int_{0}^{T} f(t)\left(K_{1}|u(t)|^{\alpha}+K_{2}\right)|u(t)| d t+\int_{0}^{T} g(t)|u(t)| d t \\
& \leq K_{1} \int_{0}^{T} f(t) d t\|u\|_{\infty}^{\alpha+1}+\left[K_{2} \int_{0}^{T} f(t) d t+\int_{0}^{T} g(t) d t\right]\|u\|_{\infty} \\
& \leq C_{42}\left(\int_{0}^{T}|\dot{u}(t)|^{p(t)} d t\right)^{\frac{\alpha+1}{p^{-}}}+C_{43}\left(\int_{0}^{T}|\dot{u}(t)|^{p(t)} d t\right)^{\frac{1}{p^{-}}}+C_{44} .
\end{aligned}
$$

Because of condition $\left(\mathrm{H}_{3}\right)$ together with (2.1), for any $u \in \widetilde{W}_{T}^{1, p(t)}$, we have

$$
\begin{aligned}
& \int_{0}^{T}\left[F_{2}(u(t))-F_{2}(0)\right] d t \\
& \quad=\int_{0}^{T} \int_{0}^{1} \frac{1}{s}\left(\nabla F_{2}(s u(t))-\nabla F_{2}(0), s \tilde{u}(t)\right) d s d t \\
& \geq-\int_{0}^{T} \int_{0}^{1} r s^{\gamma-1}|u(t)|^{\gamma} d s d t \\
& \geq-\frac{r T}{\gamma}\|u\|_{\infty}^{\gamma} \\
& \geq-C_{45}\left(\int_{0}^{T}|\dot{u}(t)|^{p(t)} d t\right)^{\frac{\gamma}{p^{-}}}-C_{46} .
\end{aligned}
$$

Therefore, using (4.1), (4.16), and (4.17), we obtain

$$
\begin{aligned}
\Phi(u)= & \widehat{M}\left(\int_{0}^{T} \frac{|\dot{u}(t)|^{p(t)}}{p(t)} d t\right)+\int_{0}^{T}[F(t, u(t))-F(t, 0)] d t \\
& +\int_{0}^{T} F(t, 0) d t \\
\geq & \frac{m_{0}}{p^{+}} \int_{0}^{T}|\dot{u}(t)|^{p(t)} d t+\int_{0}^{T}\left[F_{1}(t, u(t))-F_{1}(t, 0)\right] d t \\
& +\int_{0}^{T}\left[F_{2}(u(t))-F_{2}(0)\right] d t+\int_{0}^{T} F(t, 0) d t \\
\geq & \frac{m_{0}}{p^{+}} \int_{0}^{T}|\dot{u}(t)|^{p(t)} d t-C_{42}\left(\int_{0}^{T}|\dot{u}(t)|^{p(t)} d t\right)^{\frac{\alpha+1}{p^{-}}} \\
& -C_{43}\left(\int_{0}^{T}|\dot{u}(t)|^{p(t)} d t\right)^{\frac{1}{p^{-}}}-C_{45}\left(\int_{0}^{T}|\dot{u}(t)|^{p(t)} d t\right)^{\frac{\gamma}{p^{-}}}-C_{47}
\end{aligned}
$$

for $u \in \widetilde{W}_{T}^{1, p(t)}$. Applying Lemma 2.4, one has

$$
\int_{0}^{T}|\dot{u}(t)|^{p(t)} d t \rightarrow+\infty \quad \text { as }\|u\| \rightarrow+\infty .
$$

So we can conclude that

$$
\Phi(u) \rightarrow+\infty \quad \text { as }\|u\| \rightarrow+\infty
$$

Thus, there exists a positive constant $\eta$, such that $\Phi(u) \geq \eta$. 
On the other hand, by condition $\left(\mathrm{H}_{6}\right)$, for $y \in \mathbb{R}^{N}$ and $\forall \varepsilon>0$, we have

$$
\Phi(y)=\int_{0}^{T} F(t, y) d t \leq(-K+\varepsilon) h^{q^{+}}(|y|) .
$$

Note that $h(|y|) \rightarrow+\infty$ as $|y| \rightarrow+\infty$. Choosing $\varepsilon$ small enough, we obtain

$$
\Phi(y) \rightarrow-\infty \quad \text { as }|y| \rightarrow+\infty .
$$

Consequently, there exists a positive constant $\rho$, such that $\left.\Phi\right|_{\partial B_{\rho} \cap \mathbb{R}^{N}} \leq \eta-1=\sigma$.

Now, the functional $\Phi$ satisfies all hypotheses of the saddle point theorem (see [28], Theorem 4.6), so $\Phi$ has at least a critical point. Therefore, problem (1.1) possesses at least one $T$-periodic solution in $W_{T}^{1, p(t)}$. The proof of Theorem 3.2 is completed.

\section{Proof of Theorem 3.7}

Now we state the abstract critical point theorem found in [29].

Lemma 5.1 ([29]) Let $X$ be a Banach space with a direct sum decomposition $X_{1} \oplus X_{2}$ with $\operatorname{dim} X_{2}<\infty$. Let $\Phi$ be a $C^{1}$ function on $X$ with $I(0)=0$, satisfying P.S. condition and assume that, for some $\delta>0$,

$$
\Phi(u) \geq 0, \quad \forall u \in X_{1},\|u\| \leq \delta
$$

and

$$
\Phi(u) \leq 0, \quad \forall u \in X_{2},\|u\| \leq \delta
$$

Assume also that $\Phi$ is bounded below and $\inf _{X} \Phi<0$. Then $\Phi$ has at least two nonzero critical points.

Proof of Theorem 3.7 According to Lemma 5.1, we claim that system (1.1) has at least two nontrivial distinct solutions in $W_{T}^{1, p(t)}$.

From the proof of Theorem 3.1, we know that $\Phi$ is coercive and bounded below. Suppose that there exists $\left\{u_{n}\right\} \subset W_{T}^{1, p(t)}$ such that $\Phi^{\prime}\left(u_{n}\right) \rightarrow 0$ as $n \rightarrow \infty$, and $\Phi\left(u_{n}\right)$ is bounded. In a way similar to the proof of Theorem 3.2, we can see that $\left\{u_{n}\right\}$ is a bounded sequence and $\left\{u_{n}\right\}$ has a convergent subsequence in $W_{T}^{1, p(t)}$, which implies that $\Phi$ satisfies the PS condition.

Now, we claim that $\Phi$ has a local linking at zero. On the one hand, in view of condition $\left(\mathrm{H}_{7}\right)$, there exist two constants $\varepsilon$ and $\rho$ such that

$$
0<\varepsilon<\min \left\{d_{0}, \frac{m_{0}}{2 p^{+} T d_{0}^{p^{+}}}\right\} \text {and } 0<\rho<\varepsilon,
$$

where $d_{0}$ is the same as in (2.3), and

$$
|F(t, u)| \leq\left(\frac{m_{0}}{2 p^{+} T d_{0}^{p^{+}}}+\varepsilon\right)|u|^{p^{+}}
$$

for a.e. $t \in[0, T]$ and $|u| \leq \rho$. 
Let $\delta_{1}:=\rho / d_{0}$, if $\|u\| \leq \delta_{1}$, by (5.1), we can see that $\delta_{1}<1$.

By virtue of condition $\left(\mathrm{M}_{0}\right)$, (2.3), (5.2), and Lemma 2.2 , for $\|u\| \leq \delta_{1}$, one has

$$
\begin{aligned}
\Phi(u) & =\widehat{M}\left(\int_{0}^{T} \frac{\mid \dot{u}(t) p^{p(t)}}{p(t)} d t\right)+\int_{0}^{T} F(t, u(t)) d t \\
& \geq \int_{0}^{\int_{0}^{T} \frac{\dot{u}(t) p^{p(t)}}{p(t)} d t} M(s) d s-\left(\frac{m_{0}}{2 p^{+} T d_{0}^{p^{+}}}+\varepsilon\right) \int_{0}^{T}|u(t)|^{p^{+}} d t \\
& \geq \frac{m_{0}}{p^{+}} \int_{0}^{T}|\dot{u}(t)|^{p(t)} d t-\left(\frac{m_{0}}{2 p^{+} T d_{0}^{p^{+}}}+\varepsilon\right) T\|u\|_{\infty}^{p^{+}} \\
& \geq \frac{m_{0}}{p^{+}}\|u\|^{p^{+}}-\left(\frac{m_{0}}{2 p^{+} T d_{0}^{p^{+}}}+\varepsilon\right) T d_{0}^{p^{+}}\|u\|^{p^{+}} \\
& =\left(\frac{m_{0}}{2 p^{+}}-T d_{0}^{p^{+}} \varepsilon\right)\|u\|^{p^{+}} .
\end{aligned}
$$

This implies that $\Phi(u) \geq 0$, for $u \in \widetilde{W}_{T}^{1, p(t)}$ with $\|u\| \leq \delta_{1}$.

On the other hand, by $\left(\mathrm{H}_{8}\right)$, for $u \in \mathbb{R}^{N}$ with $|u|=\|u\| \leq \delta_{2}$, we have

$$
\begin{aligned}
\Phi(u) & =\widehat{M}\left(\int_{0}^{T} \frac{|\dot{u}(t)|^{p(t)}}{p(t)} d t\right)+\int_{0}^{T} F(t, u(t)) d t \\
& =\int_{0}^{T} F(t, u(t)) d t \\
& \leq 0 .
\end{aligned}
$$

Let $\delta \leq \min \left\{\delta_{1}, \delta_{2}\right\}$, hence $\Phi$ has a local linking at zero with respect to the space decomposition $W_{T}^{1, p(t)}=\widetilde{W}_{T}^{1, p(t)} \oplus \mathbb{R}^{N}$.

If $\inf _{W_{T}^{1, p(t)}} \Phi(u)<0$, all conditions in Lemma 5.1 are fulfilled. Hence, it follows from Lemma 5.1 that system (1.1) has at least two distinct solutions in $W_{T}^{1, p(t)}$.

If $\inf _{W_{T}^{1, p(t)}} \Phi(u) \geq 0$, according to (5.4) one has

$$
\Phi(x)=\inf _{W_{T}^{1, p(t)}} \Phi=0
$$

for all $x \in \mathbb{R}^{N}$ with $\|x\| \leq \delta$, which implies that all $x \in \mathbb{R}^{N}$ with $\|x\| \leq \delta$ are minima of $\Phi$. That is to say, system (1.1) has infinitely many solutions in $W_{T}^{1, p(t)}$.

\section{Proof of Theorem 3.8}

The proof relies on the following lemma.

Lemma 6.1 ([30]) Let $E$ be an infinite dimensional Banach space and let $\Psi \in C^{1}(E, \mathbb{R})$ be even, satisfy $(P S)$, and $\Psi(0)=0$. If $E=E_{1} \oplus E_{2}$, where $E_{1}$ is finite dimensional, and $\Psi$ satisfies:

$\left(\Psi_{1}\right) \quad \Psi$ is bounded from above on $E_{2}$.

$\left(\Psi_{2}\right)$ For each finite dimensional subspace $\widetilde{E} \subset E$, there are positive constants $\rho=\rho(\widetilde{E})$ and $\sigma=\sigma(\widetilde{E})$ such that $\Psi \geq 0$ on $B_{\rho} \cap \widetilde{E}$ and $\left.\Psi\right|_{\partial B_{\rho} \cap \widetilde{E}} \geq \sigma$ where $B_{\rho}=\{x \in E ;\|x\| \leq \rho\}$.

Then $\Psi$ possesses infinitely many nontrivial critical points. 
Proof of Theorem 3.8 The proof relies on Lemma 6.1. We will consider the functional

$$
\Psi(u)=-\Phi(u)=-\widehat{M}\left(\int_{0}^{T} \frac{|\dot{u}(t)|^{p(t)}}{p(t)} d t\right)-\int_{0}^{T} F(t, u(t)) d t .
$$

Then it is easy to see that the critical point of $\Psi$ is still the solution of system (1.1). Condition $\left(\mathrm{H}_{9}\right)$ implies $\Psi(0)=0$ and $\Psi(u)=\Psi(-u)$. Similar to the argument of Theorem 3.2, we see that $\Psi$ is bounded from above on $\widetilde{W}_{T}^{1, p(t)}$ and $\Psi$ satisfies (PS) condition on $W_{T}^{1, p(t)}$.

The above discussion shows that we only need to verify condition $\left(\Psi_{2}\right)$ of Lemma 6.1. For each finite dimensional subspace $\widetilde{E} \subset W_{T}^{1, p(t)}$, all norms on $\widetilde{E}$ are equivalent. Hence, there exist positive constants $d_{1}$ and $d_{2}$ such that

$$
d_{1} \int_{0}^{T}|\dot{u}(t)|^{p(t)} d t \leq\|u\|^{p^{-}} \leq d_{2}\left(\int_{0}^{T}|u(t)|^{\eta p^{-}} d t\right)^{\frac{1}{\eta}} .
$$

Let $s_{1}$ is an arbitrary positive constant, by condition $\left(\mathrm{M}_{2}\right)$ we have

$$
\frac{M(s)}{\widehat{M}(s)} \leq \frac{\eta}{s}
$$

for every $s \in\left[s_{1},+\infty\right)$, where $\widehat{M}(s)=\int_{0}^{s} M(\sigma) d \sigma$. Integrating this inequality we obtain

$$
\int_{s_{1}}^{s} \frac{M(\sigma)}{\widehat{M}(\sigma)} d \sigma=\ln \frac{\widehat{M}(s)}{\widehat{M}\left(s_{1}\right)} \leq \int_{s_{1}}^{s} \frac{\eta}{\sigma} d \sigma=\ln \left(\frac{s}{s_{1}}\right)^{\eta}
$$

for every $s \in\left[s_{1},+\infty\right)$. Therefore,

$$
\widehat{M}(s) \leq \frac{\widehat{M}\left(s_{1}\right)}{s_{1}^{\eta}} s^{\eta}
$$

for every $s \in\left[s_{1},+\infty\right)$. Thus there exist constants $m_{1}>0$ and $m_{2}>0$, such that

$$
\widehat{M}(s) \leq m_{1} s^{\eta}+m_{2}
$$

for all $s>0$, where $m_{1}:=\frac{\widehat{M}\left(s_{1}\right)}{s_{1}^{\eta}}$ and $m_{2}:=\max _{s \in\left[0, s_{1}\right]} \widehat{M}(s)$.

It follows from $\left(\mathrm{H}_{10}\right)$ that there exist positive constants $\vartheta$ and $L$, such that

$$
\left[-\frac{m_{1}}{\left(d_{1} p^{-}\right)^{\eta}}+\frac{\vartheta}{\left(d_{2}\right)^{\eta}}\right]\left(\frac{L}{C_{0}}\right)^{\eta p^{-}}-m_{2}>0
$$

and

$$
F(t, u) \leq-\vartheta|u|^{\eta p^{-}}
$$

for $u \in W_{T}^{1, p(t)}$ with $|u| \leq L$ and a.e. $t \in[0, T]$.

By (6.1), (6.2), and (6.3), for $u \in \widetilde{E}$ with $\|u\| \leq \frac{L}{C_{0}}$, we have

$$
\begin{aligned}
\Psi(u) & =-\widehat{M}\left(\int_{0}^{T} \frac{|\dot{u}(t)|^{p(t)}}{p(t)} d t\right)-\int_{0}^{T} F(t, u(t)) d t \\
& \geq-m_{1}\left(\int_{0}^{T} \frac{|\dot{u}(t)|^{p(t)}}{p(t)} d t\right)^{\eta}-m_{2}+\vartheta \int_{0}^{T}|u(t)|^{\eta p^{-}} d t
\end{aligned}
$$




$$
\begin{aligned}
& \geq-\frac{m_{1}}{\left(p^{-}\right)^{\eta}}\left(\int_{0}^{T}|\dot{u}(t)|^{p(t)} d t\right)^{\eta}-m_{2}+\vartheta \int_{0}^{T}|u(t)|^{\eta p^{-}} d t \\
& \geq\left[-\frac{m_{1}}{\left(d_{1} p^{-}\right)^{\eta}}+\frac{\vartheta}{\left(d_{2}\right)^{\eta}}\right]\|u\|^{\eta p^{-}}-m_{2} .
\end{aligned}
$$

Let $\rho=\frac{L}{C_{0}}$ and $\sigma=\left[-\frac{m_{1}}{\left(d_{1} p^{-}\right)^{\eta}}+\frac{\vartheta}{\left(d_{2}\right)^{\eta}}\right]\left(\frac{L}{C_{0}}\right)^{\eta p^{-}}-m_{2}$. Thus, condition $\left(\Psi_{2}\right)$ holds.

Now, the functional $\Psi$ satisfies all hypotheses of Lemma 6.1, so $\Psi$ possesses infinitely many nontrivial critical points. Thus the proof is finished.

\section{Competing interests}

The author declares that they have no competing interests.

\section{Author's contributions}

The author read and approved the final manuscript.

\section{Acknowledgements}

This work is supported by the National Natural Science Foundation of China (31260098).

Received: 1 September 2016 Accepted: 18 November 2016 Published online: 25 November 2016

\section{References}

1. Mawhin, J, Willem, M: Critical Point Theory and Hamiltonian Systems. Springer, New York (1989)

2. Wang, $Z Y$, Zhang, JH: Periodic solutions of a class of second order non-autonomous Hamiltonian systems. Nonlinear Anal., Theory Methods Appl. 72, 4480-4487 (2010)

3. $\mathrm{Wu}, \mathrm{Y}, \mathrm{An}, \mathrm{TQ}$ : Existence of periodic solutions for non-autonomous second-order Hamiltonian systems. Electron. J. Differ. Equ. 2013, 77 (2013)

4. Meng, Q, Tang, XH: Solutions of a second-order Hamiltonian system with periodic boundary conditions. Commun. Pure Appl. Anal. 9, 1053-1067 (2010)

5. Wang, DB, Yang, K: Existence of periodic solutions for a class of second order Hamiltonian systems. Bound. Value Probl. 2005, 199 (2015)

6. Tang, CL: Periodic solutions for non-autonomous second-order systems with sublinear nonlinearity. Proc. Am. Math. Soc. 126, 3263-3270 (1998)

7. Tang, CL, Ye, YW: Periodic solutions for some nonautonomous second order Hamiltonian systems. J. Math. Anal. Appl. 344, 462-471 (2008)

8. Tang, $\mathrm{CL}, \mathrm{Wu}, \mathrm{XP}$ : Periodic solutions for a class of new superquadratic second order Hamiltonian systems. Appl. Math. Lett. 34, 65-71 (2014)

9. Pipan, J, Schechter, M: Non-autonomous second order Hamiltonian systems. J. Differ. Equ. 257, 351-373 (2014)

10. Li, L, Schechter, M: Existence solutions for second order Hamiltonian systems. Nonlinear Anal., Real World Appl. 27, 283-296 (2016)

11. Pasca, D, Tang, CL: Periodic solutions of non-autonomous second order systems with $(q(t), p(t))$-Laplacian. Math. Slovaca 64, 913-930 (2014)

12. Fan, XL, Fan, X: A Knobloch-type result for $p(t)$-Laplacian systems. J. Math. Anal. Appl. 282, 453-464 (2003)

13. Wang, $X J$, Yuan, R: Existence of periodic solutions for $p(t)$-Laplacian systems. Nonlinear Anal., Theory Methods Appl. $70,866-880$ (2009)

14. Ge, B, Xue, XP, Zhou, QM: Existence of periodic solutions for a differential inclusion systems involving the p(t)-Laplacian. Acta Math. Sci. 31, 1786-1802 (2011)

15. Zhang, L, Tang, XH, Chen, J: Infinitely many periodic solutions for some second-order differential systems with $p(t)$-Laplacian. Bound. Value Probl. 2011, 33 (2011)

16. Zhang, L, Tang, XH: Subharmonic solutions for some nonautonomous Hamiltonian systems with $p(t)$-Laplacian. Bull. Belg. Math. Soc. Simon Stevin 18, 385-400 (2011)

17. Zhang, L, Chen, Y: Existence of periodic solutions of $p(t)$-Laplacian systems. Bull. Malays. Math. Sci. Soc. (2) 35, 25-38 (2012)

18. Zhang, L, Tang, XH: Periodic solutions for some nonautonomous $p(t)$-Laplacian Hamiltonian systems. Appl. Math. 58, 39-61 (2013)

19. Chen, $\mathrm{P}$, Tang, XH, Agarwal, R: Existence of homoclinic solutions for $p(n)$-Laplacian Hamiltonian systems on Orlicz sequence space. Math. Comput. Model. 55, 989-1002 (2012)

20. Chen, $\mathrm{P}$, Tang, XH, Agarwal, R: Infinitely many homoclinic solutions for nonautonomous $p(t)$-Laplacian Hamiltonian systems. Comput. Math. Appl. 63, 751-763 (2012)

21. Qin, $B, C$ Chen, P: Existence and multiplicity of homoclinic solutions for $p(t)$-Laplacian systems with subquadratic potentials. Electron. J. Differ. Equ. 2014, 111 (2014)

22. Chipot, M, Lovat, B: Some remarks on nonlocal elliptic and parabolic problems. Nonlinear Anal., Theory Methods Appl. 30, 4619-4627 (1997)

23. Fan, XL: On nonlocal $p(x)$-Laplacian Dirichlet problems. Nonlinear Anal., Theory Methods Appl. 72, 3314-3323 (2010)

24. Guo, EL, Zhao, PH: Existence and multiplicity of solutions for nonlocal $p(x)$-Laplacian equations with nonlinear Neumann boundary conditions. Bound. Value Probl. 2012, 1 (2012)

25. Dai, GW, Ma, RY: Solutions for a $p(x)$-Kirchhoff type equation with Neumann boundary data. Nonlinear Anal., Real World Appl. 12, 2666-2680 (2011) 
26. Cammaroto, F, Vilasi, L: Multiple solutions for a Kirchhoff-type problem involving the $p(x)$-Laplacian operator. Nonlinear Anal., Theory Methods Appl. 74(5), 1841-1852 (2011)

27. Cammaroto, F, Vilasi, L: Multiplicity results for a Neumann boundary value problem involving the $p(x)$-Laplacian. Taiwan. J. Math. 16(2), 621-634 (2012)

28. Rabinowitz, PH: Minimax Methods in Critical Point Theory with Applications to Differential Equations. CBMS, vol. 65. Am. Math. Soc., Providence (1986)

29. Brezis, H, Nirenberg, L: Remarks on finding critical points. Commun. Pure Appl. Math. 44, 939-963 (1991)

30. Ding, YH: Existence and multiplicity results for homoclinic solutions to a class of Hamiltonian systems. Nonlinear Anal., Theory Methods Appl. 25(1), 1095-1113 (1995)

Submit your manuscript to a SpringerOpen ${ }^{\circ}$ journal and benefit from:

- Convenient online submission

- Rigorous peer review

- Immediate publication on acceptance

- Open access: articles freely available online

- High visibility within the field

- Retaining the copyright to your article 